\title{
RECONSTRUCTION OF SPATIALLY ORIENTATED MYOTUBES IN VITRO USING ELECTROSPUN, PARALLEL MICROFIBRE ARRAYS
}

\author{
Alexander Huber ${ }^{1}$, Andy Pickett ${ }^{2}$, and Kevin M. Shakesheff ${ }^{1 *}$ \\ ${ }^{1}$ Wolfson Centre for Stem Cells, Tissue Engineering and Modelling, The Centre for Biomolecular Sciences, School \\ of Pharmacy, University of Nottingham, Nottingham, RG7 2RD, UK \\ ${ }^{2}$ Ipsen Biopharm Ltd., Ash Road North, Wrexham, LL13 9UF, UK.
}

\begin{abstract}
The stable culture of myogenic cells and their differentiation into myotubes in vitro is often hindered by the mechanical destabilisation of the spontaneously contractile neotissue formed, resulting in the complete loss of differentiating myotubes. Electrospun, parallel aligned nylon 6/6 microfibre arrays were use successfully for the culture of C2C12 myoblasts and their differentiation to form mechanically stable, orientated myotubes in vitro. Myoblasts adhered strongly to the parallel fibre array, forming a compact cell sheath across the entire array, aligning individual cells in parallel to the direction of the fibrous substratum. The myogenic potential of $\mathrm{C} 2 \mathrm{C} 12$ myoblasts was not impaired and resulted in the formation of elongated myotubes expressing $\alpha$-actinin, adult myosin heavy chain and nicotinic acetylcholine receptors as musclespecific marker proteins. Newly formed $\mathrm{C} 2 \mathrm{C} 12$ myotubes were themselves orientated in parallel to the direction of the underlying fibrous substratum and exhibited a high level of structural integration with the surrounding cells. In contrast, non-woven, non-orientated nylon 6/6 meshes, produced by conventional electrospinning, exhibited greatly reduced levels of $\mathrm{C} 2 \mathrm{C} 12$ myoblast attachment and adherent myoblasts did not differentiate into myotubes. In conclusion, parallel microfibre arrays provided a superior microscale topography for the stable maintenance and differentiation of myotubes in vitro.
\end{abstract}

Key Words: electrospinning, nylon 6/6, scaffold, nonwoven fabric, microstructure, cell culture, C2C12 myoblast, cell adhesion, differentiation, muscle

*Address for correspondence:

Kevin M. Shakesheff

Wolfson Centre for Stem Cells,

Tissue Engineering and Modelling,

The Centre for Biomolecular Sciences,

School of Pharmacy,

University of Nottingham,

Nottingham, RG7 2RD, UK

FAX Number: 0044 (0)1159515122

E-mail: kevin.shakesheff@nottingham.ac.uk

\section{Introduction}

The careful design of the cell type-specific culture conditions has facilitated the successful maintenance and expansion of numerous cell types in vitro, including established cell lines and ex vivo cell isolates. The selection of the correct cell culture medium, in combination with an appropriate culture substratum, have been found to be essential in providing a biomimetic in vivo-like culture environment. While research into novel scaffold architectures and bioreactors has lead to the development of 3-dimensional cell culture environments, routine cell culture generally employs a 2-dimensional geometry using chemically modified polymer surfaces or biologically precoated tissue culture surfaces to facilitate cellular adhesion. Additionally, cells have been grown on chemically modified surfaces, various surface topographies and micro- or nanoenvironments to control their attachment to the provided substratum and maintain their characteristic phenotypes in various developmental stages and avoid cellular trans- and dedifferentiation from their original cell type (Falconnet et al., 2006; Khademhosseini et al., 2006; Kriparamanan et al., 2006; Yim and Leong, 2005). Spatial cues have also been used to direct cell attachment to specific sites, which can be used in the reconstruction of orientated small tissue constructs in vitro (Khademhosseini et al., 2006).

Culture and differentiation of myoblasts to form mature myotubes in vitro has been a valuable tool in the characterisation of the cellular events during myogenesis (Chen and Goldhamer, 2003; Ludolph and Konieczny, 1995). Primary myoblasts, as well as established cell lines (e.g., $\mathrm{C} 2 \mathrm{C} 12$ ), have readily been used in the reconstruction of functional myotubes in vitro and the use of topographical cues from the tissue culture substrate has facilitated the reconstruction of spatially orientated myotubes (Charest et al., 2007; Neumann et al., 2003; Riboldi et al., 2007). However, successful long term culture of fully differentiated myotubes is often hindered by an increase in the level of fibre contraction, often leading to cellular detachment from the culture surface. Several strategies have been applied to reduce the number of mature myotubes detaching from the culture surface during culture, for example embedding the cell cultures in extracellular matrix proteins, e.g. collagen, or the use of a fibroblast feeder layer (Cooper et al., 2004; Vandenburgh et al., 1988). It is thought that either treatment provides a more elastic substratum to the myoblast culture preventing cellular detachment during myogenesis. However, neither treatment allows the 


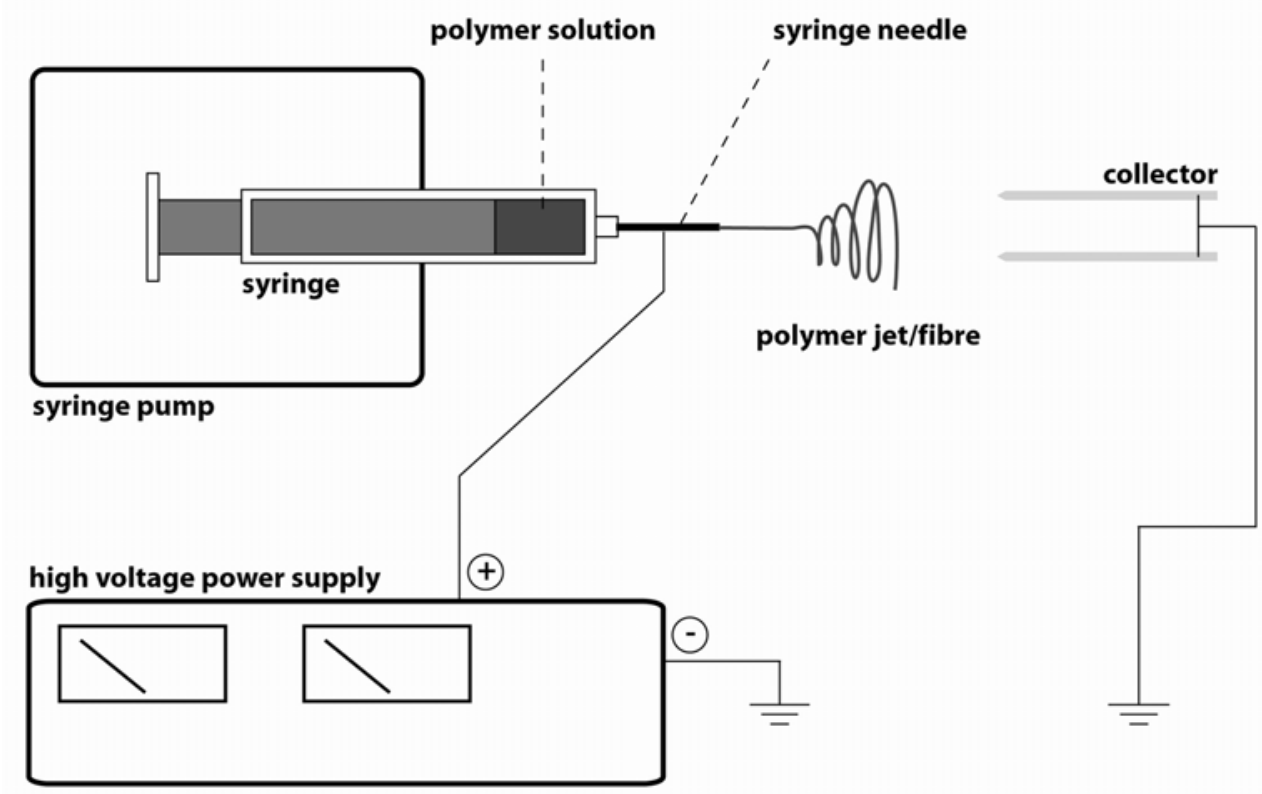

Figure 1: Schematic representation of bipolar electrospinning apparatus for the production of parallel aligned polymer fibres. A syringe pump is used to deliver the polymer solution $(30 \% \mathrm{w} / \mathrm{v}$ in formic acid) to the syringe needle at a constant rate of $100 \mu \mathrm{l} / \mathrm{min}$. A high voltage supply is used to create an electric field with a field strength of $17.5 \mathrm{kV}$ between the syringe needle and the grounded collector which consists of two grounded rods (bipolar collector) at a distance of $10 \mathrm{~cm}$.

controlled reconstruction of spatially orientated myotubes in vitro.

Fibrillar polymer surfaces produced by electrospinning have previously been suggested as a valuable tool in the construction of a more in vivo-like cell culture environment facilitating tissue reconstruction in vitro (Boudriot et al., 2006; Pham et al., 2006). Electrospinning uses a strong electric field applied to a polymer solution or polymer melt to form polymer fibres by projecting a charged polymer solution onto a collector counter electrode. The properties of the polymer fibres are predominantly controlled by the concentration and conductivity of the spun polymer solution, its delivery rate, the collector distance and the strength of the electric field. Conventional electrospinning using a flat collector plate as a counter electrode leads to the formation of a non-woven, non-orientated polymer fibre mesh (Subbiah et al., 2004). However, patterned counter electrodes or the use of a rotating collector drum or disk have been used previously in the production of fibrous polymer scaffolds with defined fibre orientations due to electrostatic forces or direct mechanical alignment (Li et al., 2005; Li et al., 2003; Tan et al., 2005).

Here, we describe the use of electrospun nylon $6 / 6$ microfilaments as part of a parallel aligned polymer microfibre array to guide the spatial reconstruction of murine $\mathrm{C} 2 \mathrm{C} 12$ myotubes in vitro without the use of additional surface modifications or a feeder layer. Nylon 6/6 microfibre arrays were constructed by electrospinning using a bipolar collector counter electrode design for the collection of parallel aligned polymer fibres. Contrary to conventionally spun non-orientated nylon 6/6 meshes, polymer scaffolds featuring a parallel fibre orientation facilitated $\mathrm{C} 2 \mathrm{C} 12$ myoblast attachment and differentiation to form structurally stable, maturating $\mathrm{C} 2 \mathrm{C} 12$ myotubes in vitro.

\section{Materials and Methods}

\section{Materials}

C2C12 myoblasts were purchased from ECACC (Salisbury, UK). Dulbecco's Modified Eagle Medium (DMEM) was supplied by Gibco Invitrogen (Paisley, UK). Dulbecco's phosphate buffered saline (PBS), L-Glutamine, heat-inactivated foetal calf serum (FCS), heat-inactivated horse serum (HS) and general antibiotic/antimycotic cell culture supplement, containing penicillin, streptomycin, and amphotericin B, were purchased from Sigma-Aldrich (Gillingham, UK).

Gill's hematoxylin type II solution was purchased from Merck (Darmstadt, Germany), Scott's tap water substitute and Eosin Y disodium salt were obtained from SigmaAldrich. Fluorescein isothiocyanate (FITC)-conjugated $\alpha$ bungarotoxin was purchased from Calbiochem (Nottingham, UK). Mouse-derived anti-mouse adult myosin heavy chain antibodies (MY-32), goat-derived antimouse $-\alpha$-actinin antibodies (H-300), and FITCconjugated anti-mouse antibodies were purchased from Autogen Bioclear (Calne, UK). Tetramethyl Rhodamine Isothiocyanate (TRITC)-conjugated anti-goat antibodies were supplied by Sigma-Aldrich and 4',6-diamidino-2phenylindole (DAPI) was purchased from Roche (Lewes, UK). Growth factor-reduced Matrigel ${ }^{\mathrm{TM}}$ was purchased from BD Biosciences (Erembodegem, Belgium). Osmium tetroxide solution and glutaraldehyde were obtained from TAAB (Reading, UK).

Nylon 6/6 (MW 262.35; $\mathrm{T}_{\mathrm{g}}=45^{\circ} \mathrm{C}$ ) was purchased from Sigma-Aldrich and polyethylene terephthalate (PET, Lumirror $\left.^{\circledR}\right)$ films were obtained from Polyester Converters Ltd. (London, UK). 
Fabrication of parallel aligned polymer fibre arrays

Parallel aligned microfibre surfaces were produced by electrospinning using a bipolar collector design (Figure 1). A $30 \%$ weight/volume (w/v) solution of nylon $6 / 6$ in formic acid with a zero shear rate viscosity of $9.5 \mathrm{~Pa} \mathrm{~s}$ was spun using an electric field of $17.5 \mathrm{kV}$ (Glassman Europe Ltd., Bramley, UK), a pump speed of $100 \mu \mathrm{l} / \mathrm{min}$ (Harvard Inst., Edenbridge, UK), and a syringe to collector distance of $10 \mathrm{~cm}$. Fibrous scaffolds were collected and anchored onto polyethylene terephthalate (PET) films. Additionally, nylon $6 / 6$ non-woven meshes were electrospun using a conventional electrospinning setup featuring a stainless steel collector plate as the counter electrode (Boudriot et al., 2006). For topographical analysis, fibrous scaffolds were sputter-coated in gold (SCD 030, Balzers Union Ltd., Balzers, Liechtenstein) and analysed using a field emission scanning electron microscope (SEM) (JEOL JSM-6060LV, JEOL (UK) Ltd., Welwyn Garden City, UK). Scaffolds used in cell culture experiments were sterilised by UVirradiation and thin-coated in growth factor-reduced Matrigel $^{\mathrm{TM}}$ (1:50) prior to cell seeding.

\section{Cell culture}

Murine $\mathrm{C} 2 \mathrm{C} 12$ myoblasts were expanded in complete growth medium (GM) consisting of DMEM supplemented with 10\% FCS, 2 mM L-Glutamine (L-Glut), 100 units/ $\mathrm{ml}$ penicillin, $0.1 \mathrm{mg} / \mathrm{ml}$ streptomycin, and $0.25 \mu \mathrm{g} / \mathrm{ml}$ amphotericin B. Cells were subcultured at $70-80 \%$ confluency using standard cell passaging techniques. Cells (passage number: 14-25) were seeded on substrates in 12well dishes and allowed to adhere for $24 \mathrm{~h}$, washed in PBS and transferred to new a 12 -well dish, exchanging the culture medium for differentiation medium (DM) consisting of DMEM supplemented with $10 \% \mathrm{HS}, 2 \mathrm{mM}$ L-Glut, 100 units $/ \mathrm{ml}$ penicillin, $0.1 \mathrm{mg} / \mathrm{ml}$ streptomycin, and $0.25 \mu \mathrm{g} / \mathrm{ml}$ amphotericin B. Myoblast differentiation was continued for 5 days replacing the culture medium every 2 days. Negative controls remained in GM for the entire culture time.

\section{Cell fixation and staining}

Tissue formation in situ was determined histologically using conventional hematoxylin and eosin $(\mathrm{H} \& \mathrm{E})$ staining. The expression of adult myosin heavy chain and $\alpha$-actinin was determined immunohistologically, while nicotinic acetylcholine receptors (nAChRs) were labeled directly using FITC-conjugated $\alpha$-bungarotoxin venom.

For H\&E staining (Gill et al., 1974; Stevens and Wilson, 1995), cultures of differentiating C2C12 myotubes grown on parallel microfibre arrays were washed in PBS and fixed in 2\% paraformaldehyde in PBS overnight at $4^{\circ} \mathrm{C}$. Samples were washed in water, incubated in Gill's hematoxylin type II solution for $15 \mathrm{~min}$, washed in tap water and blued in Scott's tap water substitute. After washing in tap water and clearing in $1 \%$ acid alcohol, the samples were incubated in $1 \%$ eosin solution for $15 \mathrm{~min}$. Samples were washed in tap water, mounted onto CryoM-Bed (Bright Instr., Huntington, UK) and snap-frozen directly in liquid nitrogen. Thin tissue sections $(3 \mu \mathrm{m})$ were cut perpendicular to the orientation of the microfibre array using a 5040 microtome (Bright Instr.). Tissue sections were transferred onto microscope slides (VWR Int., Leuven, Belgium) and analysed by light microscopy.

For immunohistology, cell cultures were washed twice in PBS and fixed in 2\% paraformaldehyde in PBS for 30 min. Samples were permeabilised in $0.1 \%$ Triton X-100 in PBS for $20 \mathrm{~min}$ and blocked in $2 \%$ bovine serum albumin in PBS for $1 \mathrm{~h}$. Samples were sequentially incubated with mouse-derived anti-mouse adult myosin heavy chain antibody (MY-32) and FITC-conjugated antimouse secondary antibody, or goat-derived anti-mouse $\alpha$-actinin antibody (H-300) and TRITC-conjugated antigoat secondary antibody. Cell nuclei were counterstained with $0.02 \%$ DAPI in PBS.

As a third marker of differentiation, cells were stained directly using FITC-conjugated $\alpha$-bungarotoxin venom to label acetylcholine receptors (McCann et al., 2006). Samples were rinsed in PBS, fixed in $2 \%$ paraformaldehyde in PBS and permeabilised in $0.1 \%$ Triton X-100. Samples were blocked in 2\% BSA in PBS, incubated in FITC-conjugated $\alpha$-bungarotoxin in PBS for $1 \mathrm{~h}$, and counterstained with DAPI.

The fluorescence staining patterns (FITC: ex/em 530/ 620 nm PI, TRITC: ex/em 500/520 nm PI, DAPI: ex/em $350 / 460 \mathrm{~nm}$ PI) were observed using an inverted light microscope (Leica Microsystems Ltd., Bensheim, Germany).

Samples were also analysed by SEM. Briefly, samples were rinsed in PBS, fixed in $2 \%$ glutaraldehyde and washed in PBS. Samples were fixed further in $1 \%$ osmium tetroxide for $2 \mathrm{hrs}$, washed in water and air dried before gold coating and analysis by SEM.

\section{Results}

\section{Fabrication of parallel microfibre arrays by electrospinning}

A modified design of the conventional electrospinning experimental setup was used to produce parallel aligned polymer fibres (Figure 1). A bipolar collector counter electrode induced the parallel alignment of spun polymer fibres due to induced electrostatic interactions resulting in the formation of a mat of parallel aligned polymer fibres (Figure 2a). Similarly, a conventional electrospinning setup, featuring a grounded steel plate as counter electrode, was used to produce non-orientated nylon meshes.

Nylon 6/6 microfibres were electrospun from a high viscosity solution, $30 \% \mathrm{w} / \mathrm{v}$ in formic acid, using a field strength of $17.5 \mathrm{kV}$, a delivery rate of $100 \mu \mathrm{l} / \mathrm{min}$, and a syringe to collector distance of $10 \mathrm{~cm}$. Polymer microfibres exhibited a "ribbon-like" appearance (Figure 2b), measuring approximately $400 \mathrm{~nm}$ across the narrow face and $1.5 \mu \mathrm{m}$ across the wide face of the fibre. Depending on the design of the collector counter electrode, electrospinning resulted in the formation of a parallel microfibre array with parallel aligned polymer fibres using the bipolar counter electrode. The use of a conventional electrospinning setup, on the other hand, results in the collection of a non-orientated, non-woven nylon mesh 
a
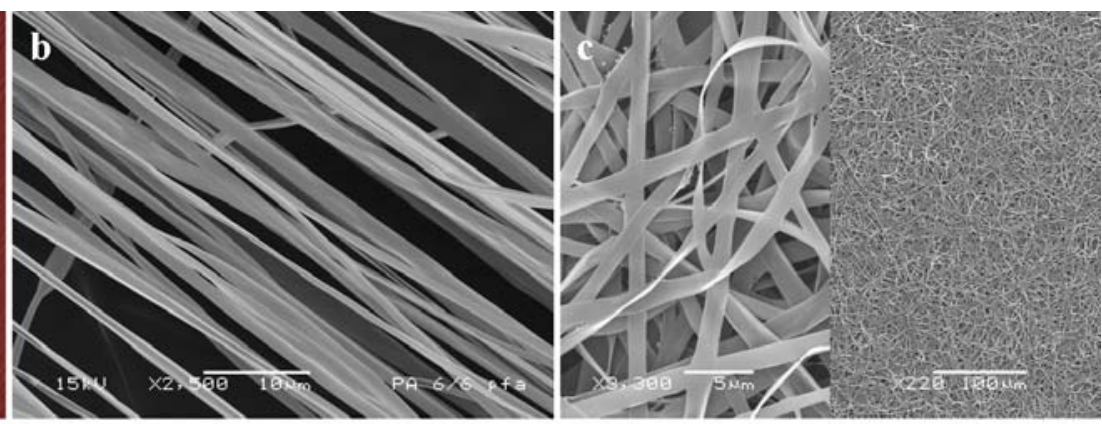

Figure 2: Electrospun, parallel aligned nylon 6/6 microfibre array and non-woven, non-orientated nylon 6/6 microfibre mesh. Light microscopic view (a; scale bar $=100 \mu \mathrm{m})$ and scanning electron micrograph (b) of a parallel aligned nylon 6/6 microfibre array produced using the bipolar collector design. Conventional electrospinning using a grounded collector shield resulted in the formation of a non-woven non-orientated nylon $6 / 6$ mesh (c).
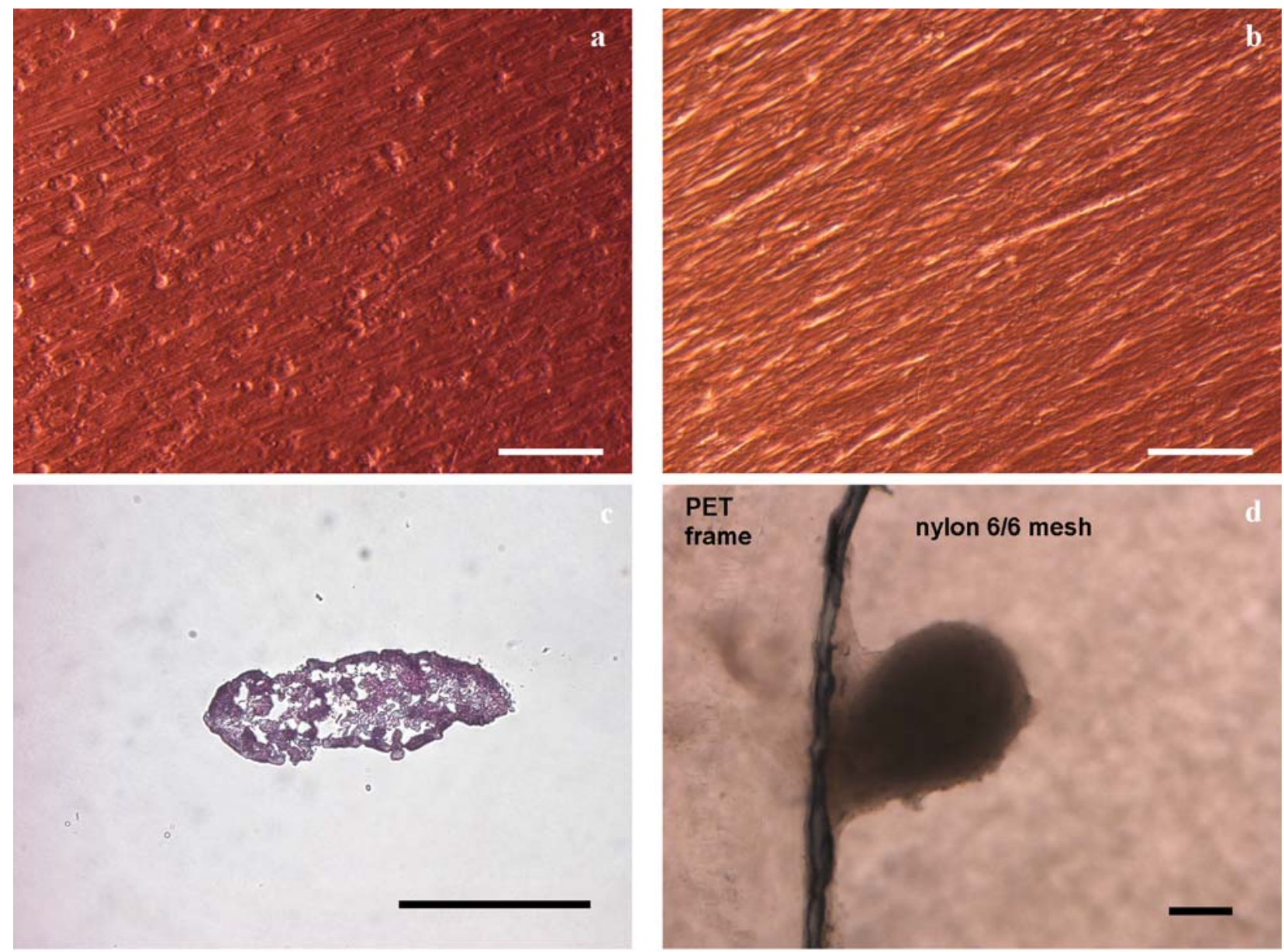

Figure 3: $\mathrm{C} 2 \mathrm{C} 12$ myotube formation on nylon 6/6 microfibre arrays. C2C12 myoblasts adhered in parallel to the direction of the underlying substratum within $24 \mathrm{~h}$ of culture (a), and differentiated within 5 days to form long myotubes that themselves aligned parallel to the underlying substratum (b). Neotissue formed across the entire parallel microfibre array, either face of the fibrous scaffold and between the individual fibres (c, H\&E staining). $\mathrm{C} 2 \mathrm{C} 12$ differentiation on non-orientated, non-woven meshes resulted in cell detachment and the formation of a large cell aggregate attached to the side of the PET frame (d); scale bars $=100 \mu \mathrm{m}$.

(Figure 2c). Parallel microfibre arrays exhibited a significantly reduced level of interfibrillar connectivity, where only few individual fibres fused with neighbouring ones to form strong crosslinks. In contrast, non-orientated, non-woven meshes formed a stiff network with numerous contact points between the individual fibres.

\section{C2C12 myotube formation on microfibre arrays}

$\mathrm{C} 2 \mathrm{C} 12$ myoblasts readily adhered to the parallel aligned microfibre array within 24 hours of culture. The myoblasts formed a solid cell sheath across the entire microfibre array and aligned in parallel to the direction of the underlying substratum (Figure 3a). The change of culture medium from growth medium to differentiation medium led to the formation of numerous elongated myotubes, approximately 200-300 $\mu \mathrm{m}$ in length, across the entire microfibre array. Again, newly formed $\mathrm{C} 2 \mathrm{C} 12$ myotubes aligned in parallel to the direction of the underlying fibrous substratum 

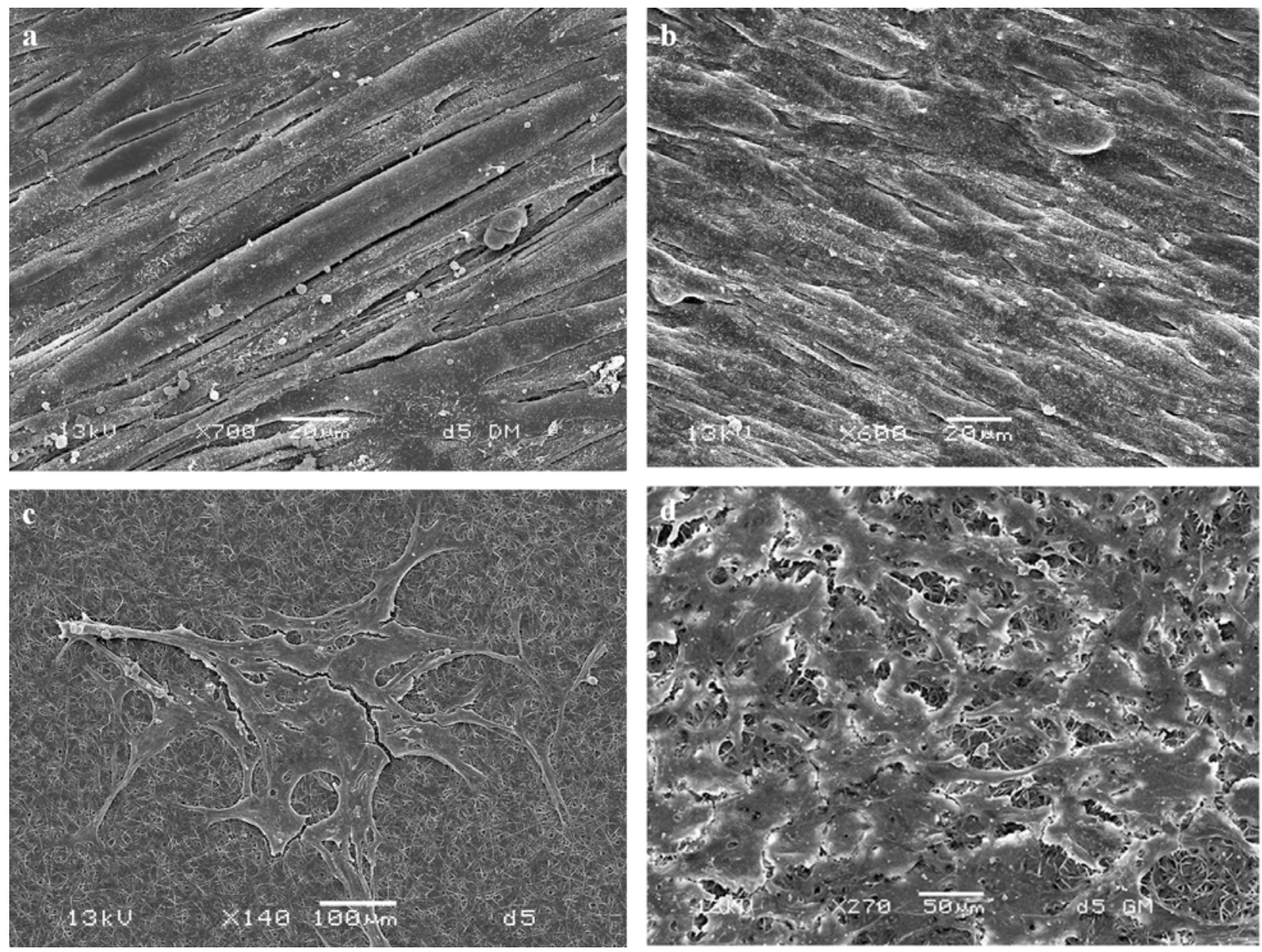

Figure 4: Scanning electron micrographs of $\mathrm{C} 2 \mathrm{C} 12$ myoblast differentiation on parallel nylon 6/6 microfibre arrays or non-woven, non-orientated nylon 6/6 microfibre meshes. On parallel nylon 6/6 microfibre arrays, C2C12 myoblasts cultures in differentiation medium differentiated within 5 days to form elongated myotubes that aligned in parallel to the direction of the underlying substratum (a). $\mathrm{C} 2 \mathrm{C} 12$ myoblasts maintained in serum-containing growth medium did not differentiate to form myotubes, but also aligned in parallel to the underlying substratum (b). Few $\mathrm{C} 2 \mathrm{C} 12$ myoblasts adhered to the non-woven, non-orientated nylon 6/6 microfibre mesh, but cells did not differentiate to form myotubes in differentiation medium (c). Cultures maintained in growth medium failed to form a confluent cell layer within the 5 days of culture $(\mathrm{d})$.

(Figure 3b). H\&E staining on thin tissue sections cut perpendicular to the orientation of the fibrous scaffold exhibited a high level of neotissue formation across the entire microfibre array (Figure 3c). Cells formed a continuous sheath across the upper and lower face of the array as well as between individual fibres. C2C12 myoblasts seeded onto non-woven non-orientated meshes however, exhibited low levels of cell attachment and the formation of a single cell aggregate directly attached to the PET frame (Figure 3d).

Cell attachment and myoblast differentiation were analysed further by SEM. Individual maturating C2C12 myotubes grown on parallel microfibre arrays appeared tubular and structurally separated from the surrounding C2C12 myoblasts (Figure 4a). Cells grown in growth medium remained in the myoblastic state aligning in parallel to the underlying substratum (Figure $4 \mathrm{~b}$ ). In contrast, $\mathrm{C} 2 \mathrm{C} 12$ myoblasts attached poorly to the conventionally spun fibre meshes leaving large parts of the mesh effectively cell free. The small number of cells that were attached, were of fibroblastic appearance and did not differentiate into myotubes (Figure 4c). The continued culture of $\mathrm{C} 2 \mathrm{C} 12$ myoblasts on fibrous nylon meshes in growth medium showed cells were proliferating, but cell proliferation did not result in the formation of a confluent cell layer across the entire scaffold within the 5 days of culture (Figure 4d).

\section{C2C12 myotube maturation on parallel microfibre arrays}

Maturating C2C12 myoblasts grown on parallel microfibre arrays were analysed for their expression of marker proteins specific for maturating muscle fibres. Cell cultures were stained directly for the presence of nicotinic acetylcholine receptors (nAChRs) using FITC-conjugated $\alpha$-bungarotoxin (McCann et al., 2006). The expression of $\alpha$-actinin and myosin heavy chain in differentiating myotubes was determined indirectly by immunohistochemistry (Harris et al., 1989; Sanger et al., 2002). Maturating, multinuclear $\mathrm{C} 2 \mathrm{C} 12$ myotubes showed an 


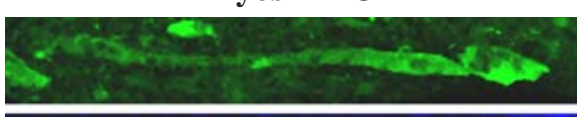

DAPI

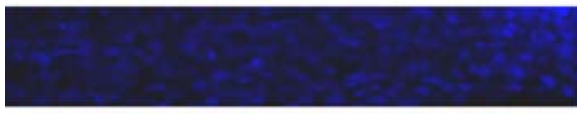

merge

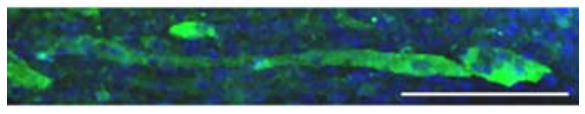

b

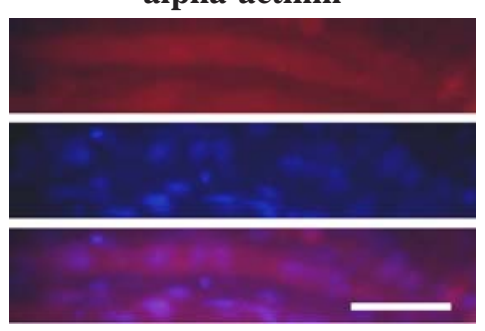

C

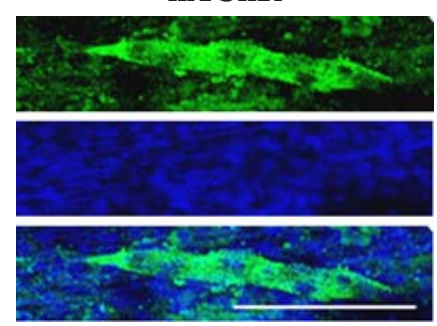

Figure 5: Expression of skeletal marker proteins in differentiating C2C12 myotubes grown on parallel aligned nylon 6/6 microfibre arrays. Maturating C2C12 myotubes exhibited an increased expression of myosin heavy chain (a; immunocytochemical staining for myosin heavy chain [top], nuclear counterstain [DAPI; middle], merged view of both [bottom], scale bar=150 $\mu \mathrm{m}), \alpha$-actinin (b; immunocytochemical staining for $\alpha$-actinin [top], nuclear counterstain [DAPI; middle], merged view of both [bottom], scale bar $=100 \mu \mathrm{m})$, and nicotinic acetylcholine receptors (c; direct staining using FITC-labeled $\alpha$-bungarotoxin [top], nuclear counterstain [DAPI; middle], merged view of both [bottom], scale bar $=150 \mu \mathrm{m})$.

increased expression of all three muscle specific marker proteins tested, i.e. myosin heavy chain, $\alpha$-actinin, and nicotinic acetylcholine receptor (Figure 5). These marker proteins are not significantly expressed in undifferentiated C2C12 myoblasts (data not shown).

\section{Discussion}

A deeper understanding of the cell type-specific growth and environmental conditions that are required by various cells types for their successful culture in vitro, has led to the development of several different scaffold geometries for use in conventional cell cultures and tissue reconstruction (Weigel et al., 2006). However, recent studies have indicated a more active relationship between cells and their culture substrata than simply providing structural support for cell attachment and proliferation. For one, chemical and structural cues have been found to be essential for the maintenance of cells in a specific developmental state during continued cell culture (Falconnet et al., 2006; Khademhosseini et al., 2006; Kriparamanan et al., 2006; Yim and Leong, 2005). On the other hand, cell culture environments need to retain a certain level of structural flexibility for the study of cellular differentiation where non-differentiated and differentiated cells require different substrata. This has been observed in the culture of myoblasts and their differentiation into contractile myotubes. While non-differentiated myoblasts can readily be grown on various stiff cell culture surfaces, differentiating myoblasts, which exhibit spontaneous contractile activity, often detach from stiff surfaces and are lost during conventional cell culture routines. Cellular embedding techniques and the use of a fibroblast feeder layer have been used previously to address this issue (Cooper et al., 2004; Vandenburgh et al., 1988).

Spatially orientated C2C12 myotubes were reconstructed on parallel microfibre arrays in vitro without the need of additional structural or cellular components. Parallel microfibre arrays were constructed by electrospinning using a bipolar collector counter electrode, which resulted in a parallel alignment of newly spun microfibres. Electrospinning of a high viscosity solution of nylon 6/6 resulted in the collection of ribbon-like fibres, a fibre type which is thought to be the result of skin formation on the projected polymer jets and the collapse of the skin connecting the opposite sides of the fibre during solvent evaporation (Koombhongse et al., 2001). Previously, ribbon-like fibre types have also been reported for the electrospinning of nylon $4 / 6$ solutions at concentrations greater than $20 \mathrm{wt} \%$ (Huang et al., 2006).

$\mathrm{C} 2 \mathrm{C} 12$ myoblasts formed a continuous sheath aligning in parallel to the direction of the underlying substratum, as observed in myogenic cell cultures on surfaces with linear topography (Baker et al., 2006; Charest et al., 2007; Evans et al., 1999; Zhong et al., 2006). Conventional polymer meshes, on the other hand, exhibited a greatly reduced level of cell adhesion. Even after five days in culture, C2C12 myoblasts grown in proliferation medium failed to reach confluency. In differentiation medium, cell proliferation was greatly reduced. Here, cells adopted a fibroblastic cell appearance and at no time fused to form myotubes, as observed in cultures using parallel microfibre arrays. Here, C2C12 myotubes differentiated to form numerous myotubes aligned in parallel to the direction of the underlying substratum. These myotubes exhibited an increased expression of three different marker proteins specific for skeletal muscle, $\alpha$-actinin, myosin heavy chain and nicotinic acetylcholine receptors. The myotube termini were deeply embedded in the adjacent cell sheath, physically anchoring the myotube to the underlying substratum. It can be assumed that the higher level of structural integration of the myotube into the existing cell sheath accounts for a higher level of stability under the increased mechanical stress caused by the spontaneous contractile activity of the newly formed myotubes which regularly results in the detachment of cells from stiff surfaces.

Parallel polymer microfibre arrays differed significantly in their level of interfibrillar connectivity when compared to the non-orientated polymer meshes produced by conventional electrospinning. Parallel polymer microfibre arrays exhibited a higher level of structural flexibility perpendicular to the direction of the 
fibrillar substratum than that observed in highly interconnected, non-woven, non-orientated electrospun polymer meshes. As a result, parallel microfibre arrays from nylon 6/6 exhibited a greatly increased level of C2C12 myoblast attachment when compared with conventional nylon $6 / 6$ meshes.

\section{Conclusion}

This study demonstrates the construction of parallel nylon $6 / 6$ microfibre arrays by electrospinning using a bifocal collector design. Parallel microfibre arrays exhibited a higher level of structural flexibility perpendicular to the axis of fibre alignment than non-woven, non-orientated nylon $6 / 6$ meshes produced by conventional electrospinning, due to a reduced level of interfibrillar connectivity. Non-orientated nylon 6/6 meshes exhibited a greatly reduced level of myoblast attachment and failed to support their differentiation into maturating myotubes. Parallel nylon 6/6 microfibre arrays, on the contrary, showed high levels of myoblast attachment. Individual cells were aligned in parallel to the direction of the underlying fibrous substratum. Myoblast differentiation led to the formation of maturating myotubes that aligned in parallel to the direction of the underlying substratum, expressing marker proteins specific to skeletal muscle.

Parallel nylon 6/6 microfibre arrays provide a superior cell culture environment for the reconstruction of spatially orientated myotubes in vitro when compared to nonorientated nylon 6/6 meshes. Additionally, parallel microfibre arrays supported the attachment of myotubes to the fibrous substratum, thereby providing a useful solution to the detachment of contractile myotubes which is observed in cultures on flat substrata.

\section{Acknowledgements}

The authors gratefully acknowledge Ipsen Biopharm Ltd. for providing a studentship to $\mathrm{AH}$.

\section{References}

Baker SC, Atkin N, Gunning PA, Granville N, Wilson K, Wilson D, Southgate J (2006) Characterisation of electrospun polystyrene scaffolds for three-dimensional in vitro biological studies. Biomaterials 27: 3136-3146.

Boudriot U, Dersch R, Greiner A, Wendorff JH (2006) Electrospinning approaches toward scaffold engineering - a brief overview. Artif Organs 30: 785-792.

Charest JL, Garcia AJ, King WP (2007) Myoblast alignment and differentiation on cell culture substrates with microscale topography and model chemistries. Biomaterials 28: 2202-2210.

Chen JC, Goldhamer DJ (2003) Skeletal muscle stem cells. Reprod Biol Endocrinol 1: 101.

Cooper ST, Maxwell AL, Kizana E, Ghoddusi M, Hardeman EC, Alexander IE, Allen DG, North KN (2004)
C2C12 co-culture on a fibroblast substratum enables sustained survival of contractile, highly differentiated myotubes with peripheral nuclei and adult fast myosin expression. Cell Motil Cytoskeleton 58: 200-211.

Evans DJ, Britland S, Wigmore PM (1999) Differential response of fetal and neonatal myoblasts to topographical guidance cues in vitro. Dev Genes Evol 209: 438-442.

Falconnet D, Csucs G, Grandin HM, Textor M (2006) Surface engineering approaches to micropattern surfaces for cell-based assays. Biomaterials 27: 3044-3063.

Gill GW, Frost JK, Miller KA (1974) A new formula for a half oxidized hematoxylin solution that neither overstains nor requires differentiation. Acta Cytol 18: 300311.

Harris AJ, Fitzsimons RB, McEwan JC (1989) Neural control of the sequence of expression of myosin heavy chain isoforms in foetal mammalian muscles. Development 107: $751-769$.

Huang C, Chen S, Lai C, Reneker DH, Qiu H, Ye Y, Hou H (2006) Electrospun polymer nanofibres with small diameters. Nanotech 17: 1558-1563.

Khademhosseini A, Langer R, Borenstein J, Vacanti JP (2006) Microscale technologies for tissue engineering and biology. Proc Natl Acad Sci U S A 103: 2480-2487.

Koombhongse S, Liu W, Reneker DH (2001) Flat Polymer Ribbons and Other Shapes by Electrospinning. J Polym Sci Part B: Polym Phys 39: 2598-2606.

Kriparamanan R, Aswath P, Zhou A, Tang L, Nguyen KT (2006) Nanotopography: cellular responses to nanostructured materials. J Nanosci Nanotechnol 6: 19051919.

Li D, Ouyang G, McCann JT, Xia Y (2005) Collecting electrospun nanofibers with patterned electrodes. Nano Lett 5: 913-916.

Li D, Wang YL, Xia YN (2003) Electrospinning of polymeric and ceramic nanofibers as uniaxially aligned arrays. Nano Lett 3: 1167-1171.

Ludolph DC, Konieczny SF (1995) Transcription factor families: muscling in on the myogenic program. FASEB J 9: 1595-1604.

McCann CM, Bracamontes J, Steinbach JH, Sanes JR (2006) The cholinergic antagonist alpha-bungarotoxin also binds and blocks a subset of GABA receptors. Proc Natl Acad Sci U S A 103: 5149-5154.

Neumann T, Hauschka SD, Sanders JE (2003) Tissue engineering of skeletal muscle using polymer fiber arrays. Tissue Eng 9: 995-1003.

Pham QP, Sharma U, Mikos AG (2006) Electrospinning of polymeric nanofibers for tissue engineering applications: a review. Tissue Eng 12: $1197-$ 1211.

Riboldi SA, Sadr N, Pigini L, Neuenschwander P, Simonet M, Mognol P, Sampaolesi M, Cossu G, Mantero S (2007) Skeletal myogenesis on highly orientated microfibrous polyesterurethane scaffolds. J Biomed Mater Res A, in press.

Sanger JW, Chowrashi P, Shaner NC, Spalthoff S, Wang J, Freeman NL, Sanger JM (2002) Myofibrillogenesis in skeletal muscle cells. Clin Orthop Relat Res: 403 Suppl: S153-162. 
Stevens A, Wilson I (1995) The hematoxylins and esosins. In Bancroft JD, Stevens A (eds) Theory and Practice of Histological Techniques. Edinburgh, Churchill Livingstone, pp 99-112.

Subbiah T, Bhat GS, Tock RW, Parameswaran S, Ramkumar SS (2004) Electrospinning of nanofibres. J Appl Polym Sci 96: 557-569.

Tan EP, Ng SY, Lim CT (2005) Tensile testing of a single ultrafine polymeric fiber. Biomaterials 26: 14531456.

Vandenburgh HH, Karlisch P, Farr L (1988) Maintenance of highly contractile tissue-cultured avian skeletal myotubes in collagen gel. In Vitro Cell Dev Biol 24: 166-174.

Weigel T, Schinkel G, Lendlein A (2006) Design and preparation of polymeric scaffolds for tissue engineering. Expert Rev Med Devices 3: 835-851.

Yim EK, Leong KW (2005) Significance of synthetic nanostructures in dictating cellular response. Nanomedicine 1: 10-21.

Zhong S, Teo WE, Zhu X, Beuerman RW, Ramakrishna S, Yung LY (2006) An aligned nanofibrous collagen scaffold by electrospinning and its effects on in vitro fibroblast culture. J Biomed Mater Res A 79: 456-463.

\section{Discussion with Reviewers}

A. Curtis: Does this type of scaffold have real or potential problems with cell access?

Authors: C2C12 myoblasts were seeded on the fibrous substrates under static conditions, in the absence of additional mechanical means, e.g. agitation. This method initially results in cell attachment to the upper face of the scaffold. However, cell differentiation and neotissue formation are not limited to this part of the scaffold, but can be observed throughout the entire scaffold (Figure 3c). This suggests that the scaffold predominantly provides a structural basis for cell attachment and alignment while possessing a low level of mechanical resistance towards cell migration. As a result, the structural rigidity of the fibres did not seem to impair cell migration and attachment to areas beyond the regions of initial cell attachment allowing cells to move freely between the individual polymer fibres to populate interfibrillar spaces and the opposite face of the scaffold.

M. Dalby: How much cell movement occurs on the nanofibers?

Authors: Individual cells exhibited a high level of motility along the entire scaffold after initial attachment, effectively populating the entire scaffold within the 24 hour seeding period before differentiation was induced. Although the movement of individual cells was not monitored as part of this research, the lack of zonal features limiting neotissue formation to distinct regions within the scaffold suggests that cells move easily between individual fibres.

M. Dalby: Do the cells mechanically tense the nanofibres? Authors: The level of mechanical contraction or stretching of individual fibres has not been part of this research. However, the contraction of the entire scaffold during neotissue formation was readily observed. The anchoring of the scaffold prevented cell-induced changes along its longitudinal axis, but led to scaffold contraction perpendicular to the direction of microfibres. The level of scaffold contraction appeared strongest towards the centre of the scaffold. 